\title{
Behavior Maps for Online Planning of Obstacle Negotiation and Climbing on Rough Terrain
}

\author{
Christian Dornhege and Alexander Kleiner
}

\section{Post Print}

N.B.: When citing this work, cite the original article.

C2007 IEEE. Personal use of this material is permitted. However, permission to reprint/republish this material for advertising or promotional purposes or for creating new collective works for resale or redistribution to servers or lists, or to reuse any copyrighted component of this work in other works must be obtained from the IEEE.

Christian Dornhege and Alexander Kleiner, Behavior Maps for Online Planning of Obstacle Negotiation and Climbing on Rough Terrain, 2007, In Proc. of the IEEE/RSJ Int. Conf. on Intelligent Robots and Systems (IROS), 3005-3011.

http://dx.doi.org/10.1109/IROS.2007.4399107

Postprint available at: Linköping University Electronic Press

http://urn.kb.se/resolve?urn=urn:nbn:se:liu:diva-72544 


\title{
Behavior maps for online planning of obstacle negotiation and climbing on rough terrain
}

\author{
Christian Dornhege and Alexander Kleiner \\ Institut für Informatik \\ University of Freiburg \\ 79110 Freiburg, Germany \\ \{dornhege, kleiner\}@informatik.uni-freiburg.de
}

\begin{abstract}
To autonomously navigate on rough terrain is a challenging problem for mobile robots, requiring the ability to decide whether parts of the environment can be traversed or have to be bypassed, which is commonly known as Obstacle Negotiation (ON). In this paper, we introduce a planning framework that extends $\mathrm{ON}$ to the general case, where different types of terrain classes directly map to specific robot skills, such as climbing stairs and ramps. This extension is based on a new concept called behavior maps, which is utilized for the planning and execution of complex skills. Behavior maps are directly generated from elevation maps, i.e. two-dimensional grids storing in each cell the corresponding height of the terrain surface, and a set of skill descriptions. Results from extensive experiments are presented, showing that the method enables the robot to explore successfully rough terrain in real-time, while selecting the optimal trajectory in terms of costs for navigation and skill execution.
\end{abstract}

\section{INTRODUCTION}

To autonomously navigate on rough terrain is a challenging problem for mobile robots, requiring the ability to decide which parts of the environment can be traversed or have to be bypassed, which is commonly known as Obstacle Negotiation (ON) [21]. While this problem has been mainly addressed for semi-rough terrain, e.g. within outdoor [13], [16], [19], and indoor scenarios [23], rough terrain, containing steep structure elements, demands robots to be explicitly aware of their context, thus allowing to execute specific skills with respect to the situation.

In this paper, we introduce a planning framework that extends ON to the general case, where different types of terrain classes directly map to specific robot skills, such as climbing stairs and ramps. The framework is based on a new concept called behavior maps, which is utilized for the planning and execution of complex skills on rough terrain. Behavior maps are directly generated from elevation maps, i.e. two-dimensional grids storing in each cell the corresponding height of the terrain surface [3], [12], and a set of skill descriptions. Skill descriptions contain a set of fuzzy rules for the classification of structures they can handle, a set of spatial constraints encoding preconditions required for their execution, a

\footnotetext{
${ }^{1}$ This research was partially supported by DFG as part of the collaborative research center SFB/TR-8 Spatial Cognition R7
}

cost function utilized for $\mathrm{A}^{*}$ planning on the map, and the skill routine to be executed. According to these skill descriptions, elevation maps are segmented into different regions, where each region corresponds to a skill that can be executed therein. We utilize Markov Random Field (MRF) models, which are automatically constructed from the set of fuzzy rules, for detecting structure elements on the elevation map. Furthermore, behavior maps are augmented with preconditions, such as starting locations and angles, which are automatically generated from the sets of spatial constraints. The resulting 2D representation encodes context information of the environment, and can efficiently be utilized for the planning and execution of skills. The final system consists of four main modules, which are all executed in real-time during navigation: elevation mapping, terrain classification, skill planning, and motion control.

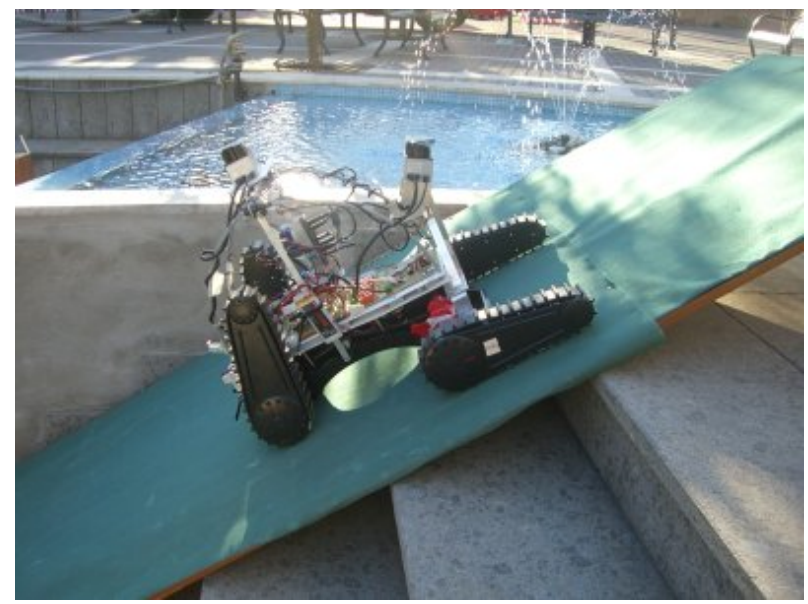

Fig. 1. The Lurker robot climbing up a ramp during the Rescue Robotics Camp 2006 in Rome.

We conducted extensive experiments within an indoor environment containing pallets and ramps, which was designed accordingly to the testing arenas build by NIST for evaluating robots for USAR (Urban Search And Rescue) [11]. Note that these kind of scenarios do not yet capture the true complexity of rough terrain found in real USAR situations. However, they provide a benchmark yearly increasing in difficulty for facilitating the stepwise development of autonomous robot mobility. 
Results from our experiments show that the robot was able to successfully explore such environments in realtime, while selecting the optimal trajectory in terms of costs for navigation and skill execution.

Former work mainly focused on behavior-based approaches for robot navigation on rough terrain. For example, Hebert and colleagues developed a tracked robot for scrambling over rubble with autonomous navigation based on visual servoing and stereo vision-based obstacle avoidance [9]. Ye and Borenstein extracted traversability field histograms from elevation maps for obstacle avoidance on a Segway platform [22]. While purely behaviorbased approaches are successful on moderately rugged 3-D terrain, they might fail within densely structured environments due to a limited lookahead needed for concluding efficient sequences of skills.

Methods for terrain classification [13], [16], [19], [18] and terrain trafficability characterization [14] have been extensively studied in the past. These methods mainly focus on the question whether a terrain feature can be traversed or has to be bypassed, e.g. they classify the terrain into classes such as road, grass, and rock.

The remainder of this paper is structured as follows. In Section II we describe the utilized hardware platform, in Section III we explain the construction of behavior maps, and in Section IV planning on these maps is described. Finally, we provide results from robot experiments in Section V and conclude in Section VI.

\section{HARDWARE PLATFORM}

Figure 1 shows the tracked Lurker robot, which is based on the Tarantula R/C toy. Although based on a toy, this robot is capable of climbing difficult obstacles, such as stairs, ramps, and random stepfields [11]. This is possible due to its tracks, which can operate independently on each side, and the "Flippers" (i.e. the four arms of the robot), which can freely be rotated at $360^{\circ}$. The base has been modified in order to enable autonomous operation. First, we added a $360^{\circ}$ freely turnable potentiometer to each of the two axes for measuring the angular position of the flippers. Second, we added 10 touch sensors to each flipper, allowing the robot to measure physical pressure when touching the ground or an object.

Furthermore, the robot is equipped with a 3 -DOF Inertial Measurement Unit (IMU) from Xsens, allowing drift-free measurements of the three Euler angles yaw, roll, and pitch, and two Hokuyo URG-X004 Laser Range Finders (LRFs), one for scan matching, and one for elevation mapping, which can be tilted in the pitch angle within $90^{\circ}$.

\section{BUILDING BEHAVIOR MAPS}

In this section we describe the process of building behavior maps, which later serve as a basis for skill planning and execution. Behavior maps are generated from elevation maps, which are two-dimensional arrays storing for each discrete location the corresponding height value and variance. We utilize a method for real-time elevation mapping on rough terrain that facilitates mapping while the robot navigates on uneven surfaces [12]. The method generates an elevation map by the successive integration of the robot's three-dimensional pose and range measurements of a downwards tilted Laser Range Finder (LRF). In order to determine the height for each location, endpoints from readings of the tilted LRF are transformed with respect to the robot's global pose, and the pitch (tilt) angle of the LRF. Thereby the threedimensional pose of the robot is tracked by integrating the orientation measured by the IMU and a translation estimate generated by a visual odometry method. Furthermore, the three-dimensional pose is updated from height observations that have been registered on the map.

Compared to conventional world representations, as for example occupancy maps, behavior maps contain context information for negotiating different kinds of structures, such as ramps and stairs. Within the proposed planning framework they serve as a basis for skill planning and execution, and are automatically generated from elevation maps and a set of skill descriptions. For this purpose skills are implementing a set of fuzzy rules for the classification of structures they can handle, a set of spatial constraints encoding preconditions required for their execution, a cost function utilized for $\mathrm{A}^{*}$ planning and the skill routine to be executed. Figure 2 summarizes the process of constructing behavior maps, and their application for planning and skill execution. Note that fuzzified features are utilized for both pre-classification and a MRF-based (Markov Random Field) classification. The pre-classification allows real-time extraction of trivially decidable regions, such as floors and walls, whereas the MRF-based classification is executed delayed in the background in order to detect undecided regions, such as ramps and stairs, more reliably. In the following, all components of skill descriptions are discussed in more detail.

\section{A. Fuzzy rules for structure detection}

Depending on the structure element to be recognized, both classification methods need to have a set of representative features that can differentiate the structure element from the environment. We choose to use fuzzified features, which are generated for each cell of the elevation map by functions that project parameters, as for example, the height difference between cells, into the $[0,1]$ interval. In contrast to binary $\{0,1\}$ features, fuzzification facilitates the continuous projection of parameters, as well as the modeling of uncertainties. This is especially useful for the later MRF classification, which requires continuous features as input. Fuzzification is carried out by combining the functions $S U p(x, a, b)$ (Equation 1) and $S \operatorname{Down}(x, a, b)$ (Equation 2), where $a$ and $b$ denote the 


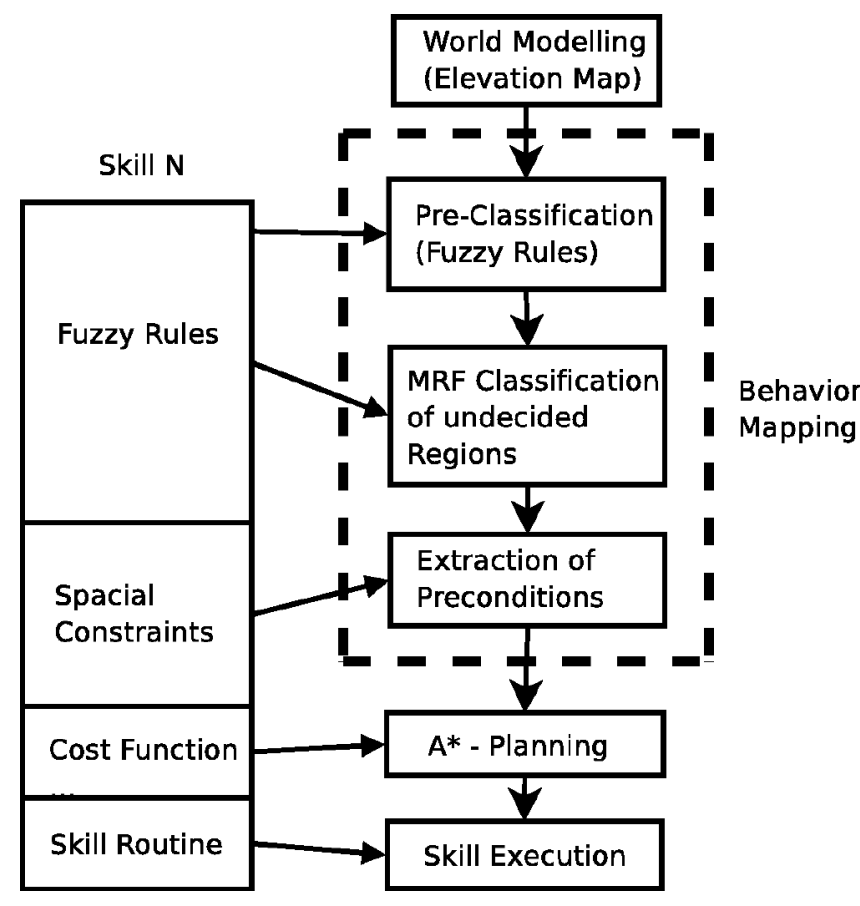

Fig. 2. The planning framework based on behavior maps, which are generated from elevation maps and skill descriptions.

desired range of the parameter.

$$
\begin{gathered}
S U p(x, a, b)= \begin{cases}0 & \text { if } x<a \\
\frac{x-a}{b-a} & \text { if } a \leq x \leq b \\
1 & \text { if } x>b\end{cases} \\
\operatorname{SDown}(x, a, b)=1-\operatorname{SUp}(x, a, b)
\end{gathered}
$$

For example, the features Flat Surface, Wall Height and Ramp Angle are build from the parameters $\delta h_{i}$, denoting the maximum height difference around a cell, and $\alpha_{i}$, denoting the angle between the normal vector $\mathbf{n}_{\mathbf{i}}$ and the upwards vector $(0,1,0)^{T}$, as shown by Equation 3 and Equation 4, respectively.

$$
\begin{array}{r}
\delta h_{i}=\max _{j \text { is neighbor to } i}\left|h_{i}-h_{j}\right| \\
\alpha_{i}=\arccos \left((0,1,0)^{T} \cdot \mathbf{n}_{\mathbf{i}}\right)=\arccos \left(n_{i_{y}}\right)
\end{array}
$$

For the described robot platform, these features are defined by:

- Flat Surface $=S \operatorname{Down}\left(\delta h_{i}, 15 \mathrm{~mm}, 40 \mathrm{~mm}\right)$

- Wall Height $=S U p\left(\delta h_{i}, 200 \mathrm{~mm}, 300 \mathrm{~mm}\right)$

- Ramp Angle $=\operatorname{SUp}\left(\alpha_{i}, 3^{\circ}, 25^{\circ}\right)$ $\operatorname{SDown}\left(\alpha_{i}, 25^{\circ}, 40^{\circ}\right)$

Each time the elevation map is updated, the preclassification procedure applies fuzzy rules on the latest height estimates in order to classify them into regions, such as flat ground, wall, and undecidable region. The rules for flat ground and wall are trivial, as for example, "if $\delta h_{i}$ is flat surface, then class is flat ground". Regions that are undecidable by the pre-processing, and which will be classified by the more time consuming MRF classifier, are extracted by "if $\delta h_{i}$ is not flat surface and $\delta h_{i}$ is not wall height, then class is undecidable region". Inference is carried out by the minimum and maximum operation, representing the logical and and or operators, respectively, whereas negations are implemented by $1-x$, following the definition given in the work of Elkan [8]. After applying the rule set to each parameter, the preclassification result is computed by defuzzification, which is carried out by choosing the rule yielding the highest output value.

The procedure is executed in real-time and is the first step to classify cells into traversable and non-traversable terrain. For discriminating more complex obstacle types, such as ramps and stairs, Markov Random Field (MRF) models, are used. Since MRFs do not linearly scale with the size of the map, they are exclusively applied on undecided regions which have not been classified by the pre-classification procedure. In order to extract those regions from the map, a color thresholding technique borrowed from computer vision [4] is utilized. This is carried out by generating a color image from the preclassified elevation map with each color corresponding to one obstacle class. Then, the image is processed by color thresholding, resulting in a segmentation of these areas into connected regions, which are then separately processed by dynamically generated MRF classifiers. An example of the result of color thresholding applied on an elevation map is shown in Figure 3.

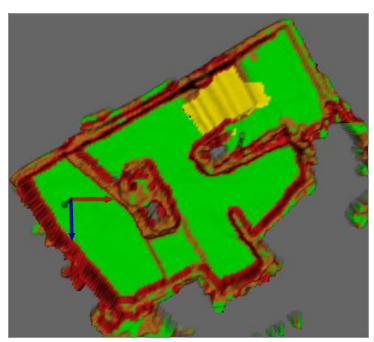

The input map

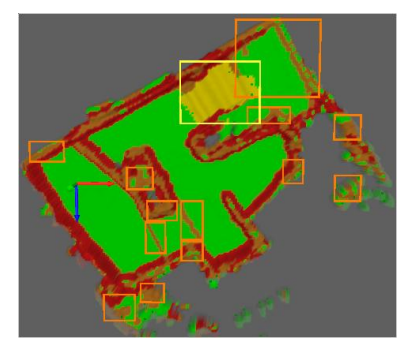

Extracted regions of interest
Fig. 3. Extraction of regions from a pre-classified map, which can be further classified by MRFs. Brown rectangles indicate obstacle regions, and yellow rectangles indicate ramp regions.

\section{B. Markov Random Fields}

To recognize complex structures from noisy data is generally a challenging task which can hardly be solved by heuristics applied on single cells of the elevation map. Hence, we utilize Markov Random Fields (MRFs) for modeling the neighborhood relations between them.

A MRF is defined by an undirected graph $\mathcal{G}=(\mathrm{Y}, \mathcal{E})$, where $\mathrm{Y}$ is a set of discrete variables $\mathrm{Y}=\left\{Y_{1}, \ldots, Y_{N}\right\}$, and $\mathcal{E}$ is a set of edges between them. Each variable $Y_{i} \in$ $\{1, \ldots, K\}$ can take on one of $K$ possible states. Hence, $\mathcal{G}$ describes a joint distribution over $\{1, \ldots, K\}^{N}$.

According to the approach of Anguelov and his colleagues [2] we utilize pairwise Markov networks, where a potential $\phi\left(y_{i}\right)$ is associated to each node and a potential $\phi\left(y_{i}, y_{j}\right)$, to each undirected edge $\mathcal{E}=\{(i j)\}(i<j)$ 
between two nodes. Consequently, the pairwise MRF model represents the joint distribution by:

$$
P_{\phi}(\mathrm{y})=\frac{1}{Z} \prod_{i=1}^{N} \phi_{i}\left(y_{i}\right) \prod_{(i j) \in \mathcal{E}} \phi_{i j}\left(y_{i}, y_{j}\right)
$$

where $Z$ denotes a normalization constant, given by $Z=$ $\sum_{\mathrm{y}^{\prime}} \prod_{i=1}^{N} \phi_{i}\left(y_{i}^{\prime}\right) \prod_{(i j) \in \mathcal{E}} \phi_{i j}\left(y_{i}^{\prime}, y_{j}^{\prime}\right)$.

A specific assignment of values to $\mathrm{Y}$ is denoted by $\mathrm{y}$ and represented by the set $\left\{y_{i}^{k}\right\}$ of $K \cdot N$ indicator variables, for which $y_{i}^{k}=I\left(y_{i}=k\right)$. In order to foster the associativity of the model, we reward instantiations that have neighboring nodes, which are labeled by the same class. This is enforced by requiring $\phi_{i j}(k, l)=$ $\lambda_{i j}^{k}$, where $\lambda_{i j}^{k}>1$, for all $k=l$, and $\phi_{i j}(k, l)=1$, otherwise [17]. Inference is carried out by solving the maximum a-posterior (MAP) inference problem, i.e. to find $\arg \max _{\mathrm{y}} P_{\phi}(\mathrm{y})$.

For our specific problem, we define the node potentials $\phi\left(y_{i}\right)$ by a vector of features, where each feature is produced by fuzzifying a parameter as defined in Section III-A. Likewise we define the edge potentials $\phi\left(y_{i}, y_{j}\right)$ by a vector of features that reflect the spatial relations between surface cells. More specific, we consider the height difference $\delta h=\left|h_{i}-h_{j}\right|$ between neighboring cells as an input parameter for features expressing those relations, as for example:

- Flat Transition $=S D o w n(\delta h, 15 \mathrm{~mm}, 40 \mathrm{~mm})$

- Obstacle Transition $=S U p(\delta h, 25 \mathrm{~mm}, 80 \mathrm{~mm})$. SDown $(\delta h, 200 \mathrm{~mm}, 350 \mathrm{~mm})$

- Wall Difference $=S U p(\delta h, 200 \mathrm{~mm}, 300 \mathrm{~mm})$

Given the node and edge potentials for each extracted region of interest, one MRF graph is dynamically constructed with each node connecting to the four closest neighbors in the vicinity. Note that the maximal number of neighbors has to be limited due to the limited computation time.

For the sake of simplicity potentials are represented by a log-linear combination $\log \phi_{i}(k)=\mathrm{w}_{n}^{k} \cdot \mathrm{x}_{i}$ and $\log \phi_{i j}(k, k)=\mathrm{w}_{e}^{k} \cdot \mathrm{x}_{i j}$, where $\mathrm{x}_{i}$ denotes the node feature vector, $\mathrm{x}_{i j}$ the edge feature vector, and $\mathrm{w}_{n}^{k}$ and $\mathrm{w}_{e}^{k}$ the row vectors according to the dimension of node features and edge features, respectively. Consequently, we can denote the MAP inference problem $\arg \max _{\mathrm{y}} P_{\phi}(\mathrm{y})$ by:

$$
\arg \max _{\mathrm{y}} \sum_{i=1}^{N} \sum_{k=1}^{K}\left(\mathrm{w}_{n}^{k} \cdot \mathrm{x}_{i}\right) y_{i}^{k}+\sum_{(i j) \in \mathcal{E}} \sum_{k=1}^{K}\left(\mathrm{w}_{e}^{k} \cdot \mathrm{x}_{i j}\right) y_{i}^{k} y_{j}^{k}
$$

Equation 6 can be solved as a linear optimization problem by replacing the quadratic term $y_{i}^{k} y_{j}^{k}$ with the variable $y_{i j}^{k}$ and adding the linear constraints $y_{i j}^{k} \leq y_{i}^{k}$ and $y_{i j}^{k} \leq y_{j}^{k}$. Hence, the linear programming formulation of the inference problem can be written as:

$$
\begin{aligned}
& \max \sum_{i=1}^{N} \sum_{k=1}^{K}\left(\mathrm{w}_{n}^{k} \cdot \mathrm{x}_{i}\right) y_{i}^{k}+\sum_{(i j) \in \mathcal{E}} \sum_{k=1}^{K}\left(\mathrm{w}_{e}^{k} \cdot \mathrm{x}_{i j}\right) y_{i j}^{k} \\
& \text { s.t. } y_{i}^{k} \geq 0, \quad \forall i, k ; \quad \sum_{k} y_{i}^{k}=1, \quad \forall i ; \\
& \quad y_{i j}^{k} \leq y_{i}^{k}, \quad y_{i j}^{k} \leq y_{j}^{k}, \quad \forall i j \in \mathcal{E}, k,
\end{aligned}
$$

which can, for example, be solved by the Simplex algorithm [7]. Furthermore, it is necessary to learn the weight vectors for the node and edge potential from data, which has been carried out by utilizing the maximum margin approach recommended by Taskar et al. [17].

\section{Encoding skill preconditions}

Due to the fact that obstacles might not be traversable from every point and every direction as flat areas are, we need to detect transitions that reliably lead the robot onto the obstacle. Furthermore, it is necessary to determine from this transitions the according preconditions for the skill execution, which are basically the starting location and starting orientation. Therefore, the user has to define for each skill the specific type of transition the skill can traverse by spacial relationships.

The Allen's Interval Calculus [1] defines qualitative relationships among one dimensional intervals by 13 base relations. Denoting the start and end points of an interval $a$ by $a^{-}$and $a^{+}$, respectively, the relations $\{$ meets, starts, finishes $\}$ can be defined by:

- $a$ meets $b:\left\{(a, b) \mid a^{-}<a^{+}=b^{-}<b^{+}\right\}$

- $a$ starts $b$ : $\left\{(a, b) \mid a^{-}=b^{-}<a^{+}<b^{+}\right\}$

- $a$ finishes $b:\left\{(a, b) \mid b^{-}<a^{-}<a^{+}=b^{+}\right\}$

Using this subset of relations, a ramp transition can be defined as $\{F$ meets $R, F$ starts $T, R$ finishes $T\}$. Figure 4(b) shows a floor-ramp transition that does not contain any other intervals besides floor $(\mathrm{F})$ and $\operatorname{ramp}(\mathrm{R})$, while $\mathrm{T}$ stands for the interval containing all intervals considered.

The clusters generated in Section III-A contain cells belonging to a specific class. To extract preconditions from these cells, the following steps are performed: First, cells that are potentially part of a transition are selected. Second, straight lines from the selected cells are extracted, where starting and ending cells of these lines are determined according to the qualitative relations describe above. In the following, these lines are termed transition edges. Third, preconditions, i.e. the exact starting locations for the skill execution, are computed.

For the extraction of suitable cells we select every cell that fulfills one of the meets rules defined by the qualitative relations. By this, cells that form a frontier from one classified region to another are selected, analogous to the definition of frontier cells [20] that define the frontier between unexplored and obstacle-free terrain within occupancy grids.

We assume that the transitions can be considered to be straight lines and apply the Hough transform [10] 
on the selected points to detect these lines. The Hough transform represents a line in normal form, so that each point $(x, y)$ is lying on the straight that has an angle $\theta$ to the $\mathrm{x}$-axis and distance $\rho$ to the origin:

$$
x \cdot \cos \theta+y \cdot \sin \theta=\rho .
$$

The transformation uses an accumulator in $(\theta, \rho)$ space to count for each point $(x, y)$ each corresponding point in $(\theta, \rho)$. Consequently, the position $\left(\theta^{*}, \rho^{*}\right)$ with the highest count represents the line that goes through most points and thus is returned as the best possible line through the point set.

In order to check whether straight lines are transition edges, the line's cells have to be considered. For each cell, this is accomplished by examining a line perpendicular to the potential transition edge and validating the spatial constraints on intervals along this line. These intervals represent adjoint cells of the same class and are constructed by concatenating adjoining cells of the same class into the same interval. If the last cell of one interval lies next to the first cell of the following interval, we consider the intervals to fulfill the meets condition. If valid cells are found over an extent of at least the robot's width, a transition edge has been detected.

Finally, the preconditions $\left(x_{s}, y_{s}, \phi_{s}\right)$ for the skill execution are calculated by projecting a starting point $\left(x_{s}, y_{s}\right)$ perpendicular from the center of the transition edge with a fixed offset in front of the obstacle. The starting direction $\phi$ is set perpendicular to the transition edge pointing towards the obstacle. Additionally, the signed height difference $h_{s}$ between the transition edge and the facing end of the obstacle, i.e. the height difference the robot has to overcome, is determined as a parameter for the skill execution. This parameter indicates, for example, whether a transition is directed upwards or downwards, and thus influences the execution of the skill.

Each map cell surrounding the transition edge will be associated with a transition edge object, which contains the transition edge's start and end point, as well as its preconditions with $h_{s}$ and the skill function, thus forming the behavior map.

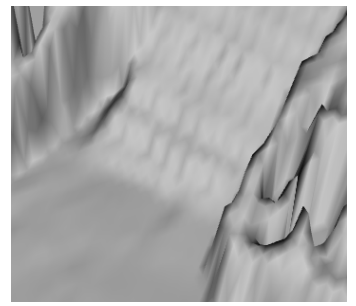

(a)

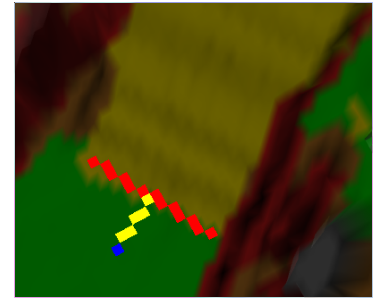

(b)
Fig. 4. Visualization of the process of determining skill preconditions: (a) Elevation map with ramp structure. (b) Classified elevation map with the detected classes floor (green), ramp (yellow), and walls (dark red), detected transition edge (red), and detected preconditions, which are the starting point (blue) and staring direction (yellow).

As an example consider the analysis of a ramp in Figure 4. Figure 4(a) shows a ramp structure on the elevation map. Figure 4(b) shows the classified elevation map with the detected classes floor (green), ramp (yellow), and walls (dark red). Constraints have been tested for each cell along a straight line through the region, resulting in the transition edge, marked in red. Note that along the transition edge the $\{F$ meets $R, F$ starts $T, R$ finishes $T\}$ rules are always valid. The detected preconditions are the starting point $\left(x_{s}, y_{s}\right)$, marked as blue, and the starting direction $\phi_{s}$ pointing upwards the ramp, marked as yellow.

\section{PLANNING AND SKILL EXECUTION}

By utilizing behavior maps for planning and skill execution we can employ two dimensional $A^{*}$ search for path planning. This is due to the fact that the behavior map itself contains the information of how to overcome an obstacle and thus there is no need to explicitly plan complex robot skills in three dimensions. The $\mathrm{A}^{*}$ algoritm performs informed search on graphs, which have a cost function assigned to their edges. To guide the search it uses a heuristic $h(n)$ to estimate the distance of a node $n$ to the goal and given this heuristic is admissable, i.e., $h(n) \leq h^{*}(n)$ for all nodes $n$, with $h^{*}$ being the optimal heuristic, $A^{*}$ guarantees the optimality of a generated path [15].

To facilitate $\mathrm{A}^{*}$ planning a graph has to be constructed from the behavior map. In a preprocessing step nontraversable cells are expanded by the robot's radius to reflect the robot's dimensions and base costs are set to 1.0 for non-traversable cells, and 0.0 for traversable cells, respectively. In a second step these costs are blurred with a Gaussian kernel to reward plans further away from walls. A cell in the behavior map represents a node in the graph and a successor function, used by $\mathrm{A}^{*}$, returns for each node $n$, those nodes, to which $n$ is connected. To permit planning over transition edges and thus generate paths over obstacles, the successor function for node $n$ returns all traversable neighbor nodes, which are either of the same class as $n$ or have a transition edge assigned to them. Note that traversability is defined only by the result of the expansion computed in the first preprocessing step, whereas the blurred costs are used solely as costs for the planning process.

Applying the $\mathrm{A}^{*}$ search to the map might lead to plans that traverse obstacles. Therefore, we need to take care of the fact that the costs for negotiating obstacles usually differ from those given by the base costs. To represent the higher amount of time spent during the skill execution on obstacles, and the increased risk of failure, a cost factor that leads to higher costs and thus to better suited planning results has to be provided by each skill.

The heuristic used for guiding the $\mathrm{A}^{*}$ search is the Euclidean distance $h=\sqrt{\delta x^{2}+\delta y^{2}}$, which is commonly employed. It is obvious that due to the increased costs when traversing obstacles this heuristic is far from being optimal, but we refrain from changing the heuristic to 
guarantee the optimality of planning. Practical experiments have shown that time needed for $\mathrm{A}^{*}$ planning constitutes no problem to the overall system.

The target selection process uses the concept of frontier cells [20], where a frontier cell is defined as being traversable and having, at least, one uninitialized neighbor. This ensures that the robot will explore unknown areas. We calculate the utility (Equation 9) based on the robots angle to the cell $\delta \theta$ and choose the frontier cell with maximum utility, which prevents frequent turning, i.e. oscillation.

$$
u=\left(\operatorname{SDown}\left(\delta \theta, 0^{\circ}, 180^{\circ}\right)\right)^{2}
$$

We want to prevent the robot from choosing plans that lead over obstacles, if there are still targets reachable by ground exploration. Therefore, the planner accepts costs for ground exploration until a certain threshold, before permitting planning over obstacles.

When executing the actual plan, the skill subroutine that has to be executed by the controller is determined from the robot's current position on the behavior map. A transition edge stores the precondition that has to be fulfilled first before executing the skill. Whenever the controller is queried to execute a new skill routine, it first ensures this precondition by turning towards the required starting direction before calling the skill itself.

The skills (besides Ground Exploration) implemented in our system use as sensory input the robot's pitch and roll from the IMU, the angles of the flippers, and touch point sensors in the flippers that can give feedback about where the flipper has contact with an obstacle. Based on features created from these sensors, a state machine is executed that can run different actions in parallel (e.g. driving forward while moving the rear flippers down). Currently our robots have the ability to lift up and drive down from a pallet-like obstacle, drive a ramp and climb up stairs.

\section{EXPERIMENTAL RESULTS}

In order to evaluate the planning framework, a fully autonomous exploration run has been conducted. Results from this run can be seen in Figure 5. The test run demonstrates the system's ability of online planning and obstacle negotiation during an exploration task on rough terrain. Figure 5(c) shows the exploration trajectory, crossing each obstacle only once, which covers the complete area. Due to the higher costs for obstacle climbing, the robot first explored flat areas before climbing over obstacles. Finally, a trajectory with a length of $23.00 \mathrm{~m}$ has been traveled within 9 minutes and 55 seconds by the robot. Main factors to the overall computation time were: the hough transform $(63.94 \mathrm{~ms} \pm 0.78 \mathrm{~ms})$, the occupancy map blur $(37.42 \mathrm{~ms} \pm 0.98 \mathrm{~ms})$ and the $\mathrm{A}^{*}$ planning $(32.26 \mathrm{~ms} \pm 4.76 \mathrm{~ms})$ on a AMD Athlon X2 $3800+$. Additionally, the MRF algorithm took $26.31 s \pm$ $27.88 \mathrm{~s}$ to classify extracted regions. Note that these computation times have been measured for an update

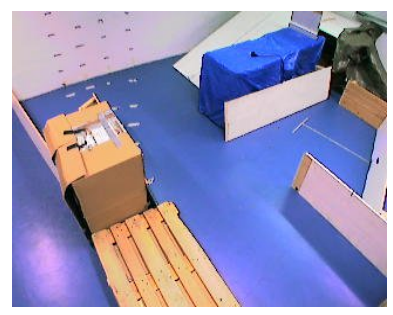

(a)

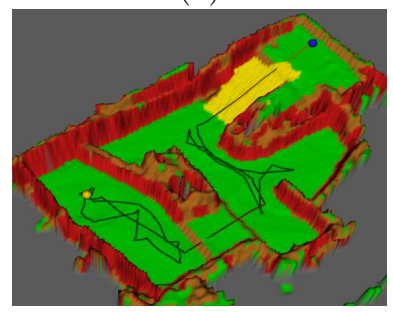

(c)

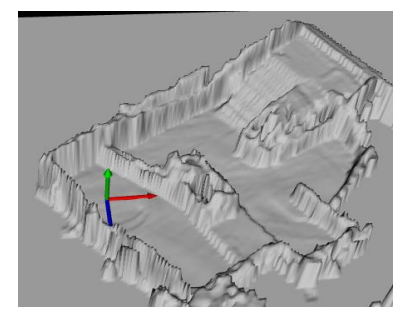

(b)

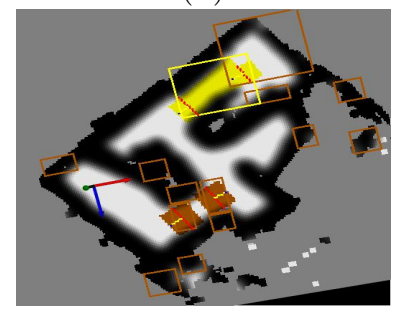

(d)
Fig. 5. (a) Test arena for the exploration task. (b) The created elevation map. (c) Classified map containing the trajectory of the exploration. (d) The behavior map showing the detected clusters of interest for obstacles (brown rectangles) and ramp (yellow rectangles) and recognized obstacle transitions (red). The robot started exploring the area at the left, crossed the pallet, continued to explore the center area and finally drove up the ramp.

of the full map consisting of 60,000 cells. During online execution, computation time is significantly lower since only map regions that were updated beforehand by the elevation mapper have to be considered. Furthermore, the comparably higher amount of time needed for the MRF classification did not limit the system's real-time ability. This is due to the fact that the robot was able to rely on the pre-classification for planning and exploration while the MRF classification has been computed timedelayed in the background.

Skills are modules that are executed with respect to the robot's context, provided by the behavior map, for climbing and obstacle negotiation. In Figure 6 a detailed view on the execution of the autonomous skill for stair climbing is presented. To achieve this task, the robot lifts itself up by pushing down with the front flippers after aligning to the stairs. Then, the robot drives forward while perceiving feedback from touchpoint sensors within the flippers if contacting the stair. This enables the robot to become aware of its state and to adjust the flipper angles accordingly. Finally, when reaching the end of the stairs, the arms are moved back into driving position. A video showing this test run and the autonomous stair climbing is available in the video proceedings [5].

\section{CONCLUSIONS AND FUTURE WORKS}

In this paper we introduced an extension to obstacle negotiation that allows robots to execute specific skills with respect to their context. The proposed planning framework is based on a new concept called behavior maps, which allows robots to deliberatively plan and execute complex skills on rough terrain. The advantage 

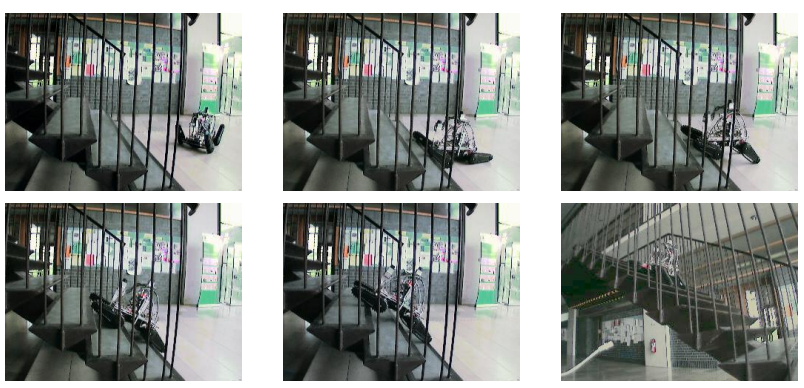

Fig. 6. Autonomous climbing of stairs.

of the described framework, i.e. the abstract definition of skills, is that it can be extended by new skills, and also be adapted to other robot types.

Our results have shown that the final system implements a truly autonomous robot that builds an elevation map of the environment, classifies this map, and plans the execution of sequences of skills. In contrast to existing related work, the proposed system performs all these steps online during execution while overcoming rough terrain. This is a much harder problem than processing data offline, e.g. to classify structures from a pre-computed elevation map captured from fixed robot locations. During online mapping, incremental states of the elevation map are only partially representing the environment due to the robot's limited field of view.

In contrast to structures found in real disaster areas, the demonstrated experiments were carried out within a simplified environment. However, we are convinced that, given reliably structure detection, the presented framework is expendable towards more complex structure components.

In future work, we will evaluate the application of learning methods within skills by exploiting the context information given by the behavior map. Furthermore, we will extend the proposed approach towards a multi-level representation of behavior maps, allowing the robot to autonomously explore multistory buildings. One target scenario in this context will be the benchmark presented by the TechX challenge [6], which will be held 2008 in Singapore.

\section{ACKNOWLEDGMENTS}

The authors gratefully acknowledge the work done by all members of the RescueRobots Freiburg team, particularly Rainer Kümmerle and Bastian Steder.

\section{REFERENCES}

[1] J. F. Allen. Maintaining knowledge about temporal intervals. Commun. ACM, 26(11):832-843, 1983.
[2] D. Anguelov, B. Taskar, V. Chatalbashev, D. Koller, D. Gupta, G. Heitz, and A.Y. Ng. Discriminative learning of markov random fields for segmentation of $3 \mathrm{D}$ range data. In IEEE Int. Conf. on Computer Vision and Pattern Recognition (CVPR), San Diego, California, June 2005.

[3] J. Bares, M. Hebert, T. Kanade, E. Krotkov, T. Mitchell, R. Simmons, and W. R. L. Whittaker. Ambler: An autonomous rover for planetary exploration. 22(6):18-22, 1989.

[4] J. Bruce, T. Balch, and M. Veloso. Fast and inexpensive color image segmentation for interactive robots. In Proceedings of IROS-2000, Japan, October 2000.

[5] C. Dornhege and A. Kleiner. Fully autonomous planning and obstacle negotiation on rough terrain using behavior maps. In Video Proc. of the IEEE/RSJ Int. Conf. on Intelligent Robots and Systems (IROS), 2007. To be published.

[6] DSTA. The techx challenge. Homepage: http://www.dsta. gov.sg/TechXChallenge/index.asp. Referenced 2007.

[7] Minieka E. and J.R. Evans. Optimization Algorithms for Networks and Graphs. CRC Press, 1992.

[8] Charles Elkan. The paradoxical success of fuzzy logic. In Proceedings of the Eleventh National Conference on Artificial Intelligence, pages 698-703, Menlo Park, California, 1993. AAAI Press.

[9] M. Hebert, R. MacLachlan, and P. Chang. Experiments with driving modes for urban robots. In Proceedings of SPIE, 1999.

[10] P.V.C. Hough. Methods and means for recognizing complex patterns. In U.S. Patent 069654, 1962.

[11] A. Jacoff, E. Messina, and J. Evans. Experiences in deploying test arenas for autonomous mobile robots. In Proc. of the PerMIS Workshop, Mexico, 2001.

[12] A. Kleiner and C. Dornhege. Real-time localization and elevation mapping within urban search and rescue scenarios. Journal of Field Robotics, 2007. Accepted for publication.

[13] R. Manduchi, A. Castano, A. Talukder, and Larry Matthies. Obstacle detection and terrain classification for autonomous off-road navigation. Auton. Robots, 18(1):81-102, 2005.

[14] L. Ojeda, J. Borenstein, and G. Witus. Terrain trafficability characterization with a mobile robot. In G. R. Gerhart, C. M. Shoemaker, and D. W. Gage, editors, Unmanned Ground Vehicle Technology VII, volume 5804, pages 235-243, 2005.

[15] Stuart J. Russell and Peter Norvig. Artificial Intelligence. A Modern Approach. Prentice-Hall, 1995.

[16] A. Talukder, R. Manduchi, R. Castano, K. Owens, L. Matthies, A. Castano, and R. Hogg. Autonomous terrain characterisation and modelling for dynamic control of unmanned vehicles. In IEEE/RSJ Int. Conf. on Intelligent Robots and Systems (IROS), pages 708-713, 2002.

[17] B. Taskar, V. Chatalbashev, and D. Koller. Learning associative markov networks. In Proc. of the 21th ICML, 2004.

[18] S. Thrun, M. Montemerlo, H. Dahlkamp, D. Stavens, A. Aron, J. Diebel, P. Fong, J. Gale, M. Halpenny, G. Hoffmann, K. Lau, C. Oakley, M. Palatucci, V. Pratt, P. Stang, S. Strohband, C. Dupont, L.-E. Jendrossek, C. Koelen, C. Markey, C. Rummel, J. van Niekerk, E. Jensen, P. Alessandrini, G. Bradski, B. Davies, S. Ettinger, A. Kaehler, A. Nefian, and P. Mahoney. Stanley, the robot that won the DARPA grand challenge. 23(9):655-656, 2006.

[19] N. Vandapel, D. Huber, A. Kapuria, and M. Hebert. Natural terrain classification using 3-d ladar data. In IEEE International Conference on Robotics and Automation, volume 5, pages 5117 - 5122, April 2004.

[20] B. Yamauchi. A frontier-based approach for autonomous exploration. In $C I R A, 1997$.

[21] C. Ye and J. Borenstein. A new terrain mapping method for mobile robot obstacle negotiation. In Proc. of the $U G V$ Technology Conf. at the 2003 SPIE AeroSense Symposium, pages 21-25, Orlando, USA, 2003.

[22] C. Ye and J. Borenstein. Obstacle avoidance for the segway robotic mobility platform. In Proc. of the American Nuclear Society Int. Conf. on Robotics and Remote Systems for Hazardous Environments, pages 107-114, Gainesville, USA, 2004.

[23] C. Ye and J. Borenstein. T-transformation: a new traversability analysis method for terrain navigation. In Proc. of the SPIE Defense and Security Symposium, Orlando, USA, 2004. 